\section{High-performance detectors for visible near-IR imaging sensors developed}

\section{The pitch}

There is significant interest in developing low-cost visible infrared (IR) sensors for a variety of applications such as imaging sensors for defense, homeland security, and commercial applications (Figure 1). There are several other technologies such as indium gallium arsenide (InGaAs), indium antimonide (InSb), and mercury cadmium telluride (HgCdTe) that cover different parts of the IR spectrum. HgCdTe IR focal-plane arrays are being developed for $3-5 \mu \mathrm{m}$ and $8-14 \mu \mathrm{m}$ applications. InSb is being used for 3-5 $\mu \mathrm{m}$ applications.

Similarly InGaAs offers an attractive approach for visible near-IR sensors that can cover spectral bandwidths up to $1.8 \mu \mathrm{m}$. Silicon germanium ( $\mathrm{SiGe}$ ) offers a low-cost alternative for developing visible near-IR sensors that will not require any cooling and can operate from 0.4 $\mu \mathrm{m}$ to $1.6 \mu \mathrm{m}$. In addition, there are new approaches that can further increase the spectral sensitivity to $2 \mu \mathrm{m}$ and beyond. This technology has the potential for a small unit cell size and can be built with megapixel visible near-IR cameras.

The characteristics that make IR sensors appropriate for defense applications also benefit many industrial and

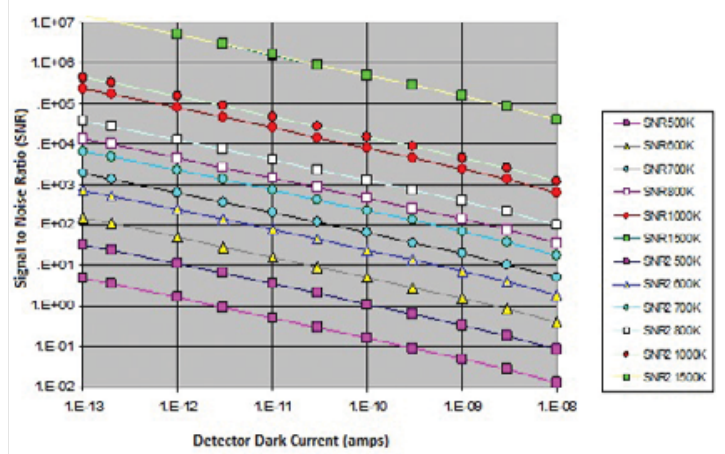

Figure 1. Signal-to-noise ratio as a function of dark current (current that flows in a photodetector when not receiving any light) for various detection target temperatures (radiances) for a SiGe detector array. scientific applications. Several applications for environmental monitoring and control use IR sensors. For example, low-cost IR-based systems can be used for night vision for vehicles, for robot vision, and for medical thermography for cancer and tumor detection during diagnosis and surgery. With higher volume and lower cost these sensors and cameras will also be used in cell phones for a variety of defense and commercial applications. The market for these sensors is projected to grow over the next decade.

\section{The technology}

SiGe-based visible-IR arrays offer a low-cost alternative for developing near-IR sensors that will not require any cooling and can operate in the visible and near-IR (VIS-NIR) bands. To efficiently access the VIS-NIR band for various applications, high-Ge-content layers are required. Magnolia Optical Technologies' SiGe pin (large intrinsic region sandwiched between $p$-doped and $n$-doped semiconductor regions) photodiodes are fabricated with $1.7-\mu \mathrm{m}$ thick Ge layers grown directly on $15 \mathrm{~cm} \mathrm{Si}$ substrates, using low-pressure chemical vapor deposition in an epitaxial growth system. After Ge growth, standard complementary metal oxide semiconductor processes are used to deposit and pattern a dielectric film to open windows to the Ge surface. Then poly-Si is deposited and implanted with phosphorus to form the $n^{+}$top contact. The samples are annealed to out-diffuse the phosphorus into the underlying Ge to form a vertical pin junction in the germanium (Figure 2). As the technology for SiGe detector arrays is developed, further development will be needed for fabricating high-density large format SiGe VIS-NIR focal-plane arrays.

The company is also exploring integrating SiGe VIS-NIR detectors with $\mathrm{Si}$ - microelectromechanical systems devices. The potential advantage of integrating SiGe devices with silicon-based bolometers is the use of the silicon-based process which makes it possible for the integration to be accomplished on large silicon wafers. Magnolia is evaluating 


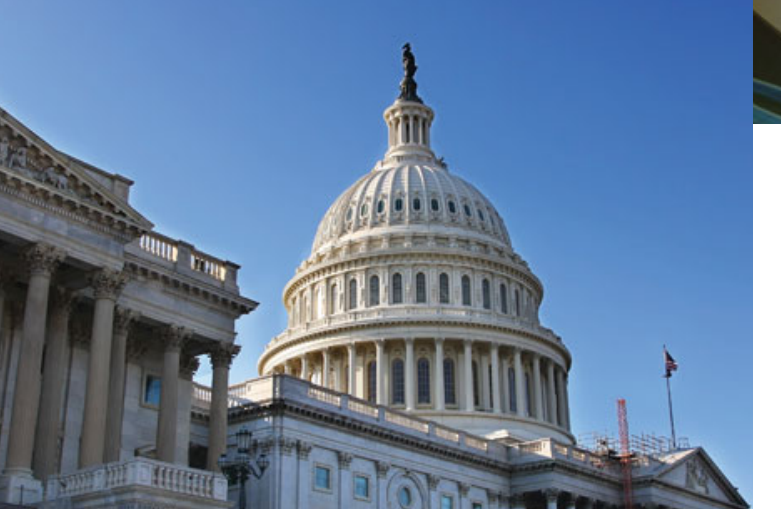

\section{Applications Sought for 2012-2013}

\section{Congressional Science and Engineering Fellowship}

\section{PROGRAM}

The Fellow spends one year working as a special legislative assistant on the staff of a member of Congress or congressional committee. Activities may involve conducting legislative or oversight work, assisting in congressional hearings and debates, and preparing briefs and writing speeches. The Fellow also attends an orientation program on congressional and executive branch operations, which includes guidance in the congressional placement process, and a year-long seminar series on science and public policy issues. These aspects of the program are administered by the American Association for the Advancement of Science.

\section{PURPOSE}

To provide Materials Research Society, The Optical Society, and The Minerals, Metals \& Materials Society members with an invaluable public policy learning experience, to contribute to the more effective use of materials and/or optical science knowledge in government, and to broaden awareness about the value of scientist and engineergovernment interaction among Society members and within the federal government.

\section{CRITERIA}

A prospective Fellow must demonstrate a record of success in research or scholarship, in a field relevant to materials science and technology and/or optical science and technology. The Fellow must also demonstrate sensitivity toward policy issues and have a strong interest in applying scientific and technical knowledge to U.S. public policy issues. The Fellow must be able to work quickly and communicate effectively on a wide variety of topics, and be able to work cooperatively with individuals having diverse viewpoints. U.S. Citizenship is not required, however, applicants must be authorized to work in the United States.

\section{AWARD}

The Fellow will have a one-year appointment beginning September 1, 2012. The Fellowship stipend will be $\$ 72,000$ plus assistance for health insurance, travel and relocation expenses to the Washington, DC area. Final selection of the Fellow will be made in early 2012.

\section{APPLICATION}

Applications must be postmarked or emailed by January 6, 2012 and must include the following materials: (1) a detailed resume, no longer than two pages, providing information about educational background, professional employment, community and professional activities, public policy and legislative experience and committee and advisory group appointments plus one additional page for professional publications and presentations; (2) a statement of approximately 1,000 words addressing the applicant's interest in the fellowship, career goals, contributions the applicant believes he or she can make as a Fellow to the legislative process and what the applicant wants to learn from the experience; and (3) three signed letters of reference specifically addressing the applicant's ability to work on Capitol Hill as a special legislative assistant.

\section{The deadline for applications is January 6, 2012.}

Letters of reference should be mailed or emailed directly by the reference to the appropriate address below and must be in PDF format, on official letterhead, and include an electronic or scanned signature. Letters of reference should not be submitted by the candidates themselves.

\section{OSA}

MRS/OSA

Congressional Science and Engineering Fellow Program

c/o Laura Kolton

The Optical Society

2010 Massachusetts Avenue, NW

Washington, DC 20036-1023

lkolton@osa.org

Applicant must be a member of MRS or OSA (or an applicant for membership) and have a doctorate.

For additional information, contact Laura Kolton at 202-416-1499, Ikolton@osa.org Donna Gillespie at 724-779-2732, gillespie@mrs.org.

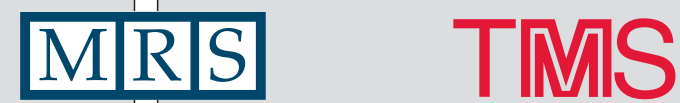

MRS/TMS

Congressional Science and Engineering Fellow Program

c/o Donna Gillespie

Materials Research Society

506 Keystone Drive

Warrendale, PA 15086

gillespie@mrs.org

Applicant must be a member of MRS or TMS

(or an applicant for membership) and have a doctorate or master's degree with three years of professional experience.

For additional information, contact

Donna Gillespie at 724-779-2732, gillespie@mrs.org or Mary Samsa at 724-814-3130, msamsa@tms.org. 